\title{
Metabolic Engineering of Histidine Kinases in Clostridium beijerinckii for Enhanced Butanol Production
}

OPEN ACCESS

Edited by:

Yi Wang,

Auburn University, United States

Reviewed by:

Petra Patakova

University of Chemistry

and Technology Prague, Czechia

Zhiqiang Wen,

Nanjing University of Science and Technology, China

Fengxue Xin,

Nanjing Tech University, China

*Correspondence:

Chuang Xue

xue.1@dlut.edu.cn

Specialty section:

This article was submitted to

Synthetic Biology,

a section of the journal

Frontiers in Bioengineering and

Biotechnology

Received: 14 January 2020

Accepted: 03 March 2020

Published: 20 March 2020

Citation:

Xin X, Cheng C, Du G, Chen L

and Xue C (2020) Metabolic

Engineering of Histidine Kinases

in Clostridium beijerinckil

for Enhanced Butanol Production.

Front. Bioeng. Biotechnol. 8:214.

doi: 10.3389/fbioe.2020.00214
Xin Xin, Chi Cheng, Guangqing Du, Lijie Chen and Chuang Xue*

School of Bioengineering, Dalian University of Technology, Dalian, China

Clostridium beijerinckii, a promising industrial microorganism for butanol production, suffers from low butanol titer and lack of high-efficiency genetical engineering toolkit. A few histidine kinases (HKs) responsible for SpoOA phosphorylation have been demonstrated as functionally important components in regulating butanol biosynthesis in solventogenic clostridia such as $C$. acetobutylicum, but no study about HKs has been conducted in C. beijerinckii. In this study, six annotated but uncharacterized candidate HK genes sharing partial homologies (no less than $30 \%$ ) with those in C. acetobutylicum were selected based on sequence alignment. The encoding region of these HK genes were deleted with CRISPR-Cas9n-based genome editing technology. The deletion of cbei2073 and cbei4484 resulted in significant change in butanol biosynthesis, with butanol production increased by 40.8 and $17.3 \%$ (13.8 g/L and $11.5 \mathrm{~g} / \mathrm{L}$ vs. $9.8 \mathrm{~g} / \mathrm{L}$ ), respectively, compared to the wild-type. Faster butanol production rates were observed, with butanol productivity greatly increased by 40.0 and $20.0 \%$, respectively, indicating these two HKs are important in regulating cellular metabolism in C. beijerinckii. In addition, the sporulation frequencies of two HKs inactivated strains decreased by 96.9 and $77.4 \%$, respectively. The other four HK-deletion (including cbei2087, cbei2435, cbei4925, and cbei1553) mutant strains showed few phenotypic changes compared with the wild-type. This study demonstrated the role of HKs on sporulation and solventogenesis in C. beijerinckii, and provided a novel engineering strategy of HKs for improving metabolite production. The hyper-butanol-producing strains generated in this study have great potentials in industrial biobutanol production.

Keywords: Clostridium beijerinckii, CRISPR-Cas9n, histidine kinases, butanol, sporulation

\section{INTRODUCTION}

During the past decades, with concerns about diminishing petroleum reserves and fluctuations in oil prices, renewable biofuels have gained intensive attentions. As a substitute for gasoline, butanol has promising physical features such as high energy density, low volatility, and less corrosivity, but clostridia-based biobutanol production is economically unfavorable due to low product titer and productivity (Xue et al., 2013; Yang et al., 2018). Clostridium beijerinckii is an important industrial microorganism which produces butanol, but is notoriously difficult to metabolically engineer and hard to break through the limitation of low product concentration (Xue et al., 2017a). Since CRISPR-Cas9 has been explored as a powerful and effective tool for genome editing of lots 
of organisms including C. beijerinckii (Li et al., 2016; Wang et al., 2016a), we expected to focus on editing the genome of C. beijerinckii based on this system.

Clostridium beijerinckii is an organism historically used for $\mathrm{ABE}$ (acetone, butanol, ethanol) fermentation. Batch $\mathrm{ABE}$ fermentation is characterized by two distinctive phases, acidogenesis and solventogenesis (Herman et al., 2017). During exponential growth, short-chain fatty acids, including acetic acid and butyric acid, are produced and accumulated in the system, which causes a drop in the culture $\mathrm{pH}$. As the culture reaches a low enough $\mathrm{pH}$, the formed acids can be re-assimilated and the culture $\mathrm{pH}$ rises. At the same time, solvent production is initiated ( $\mathrm{Li}$ et al., 2011). The metabolic transition from acidogenesis to solventogenesis is consistent with the initiation of the complex sporulation process. During sporulation, a starch-like carbohydrate called granulose accumulates in the form of a swollen, bright-phase Clostridium bacterium, in which the endospore begins to develop. Further morphological development produces free-spores, heat- and chemical-resistant cell types that do not contribute to solvent production (Al-Hinai et al., 2015; Herman et al., 2017).

Histidine kinases (HKs) are involved in perceiving and transducing environmental signals to trigger multiple cellular responses, such as cell division (Jacobs et al., 1999), nitrogen metabolism (Loomis et al., 1997), antibiotic resistance (Wilke et al., 2015) and sporulation (Xue and Cheng, 2019). Orphan HKs are HKs lacking an adjoining response regulator, as is typical for most HKs in prokaryotes (Steiner et al., 2011). Several orphan HKs, regulating spore development in C. perfringens (Hiscox et al., 2013), C. difficile (Underwood et al., 2009), C. botulinum (Wörner et al., 2006) and C. thermocellum (Mearls and Lynd, 2014), etc., have been identified. Especially, orphan HKs directly phosphorylate Spo0A, which is the master regulator of both sporulation and solventogenesis in C. acetobutylicum (Steiner et al., 2011). Spo0A directly controls the genes with the presence of one or more "0A boxes" (TGNCGAA) in their 5 ' regulatory regions (Wilkinson et al., 1995). The "0A boxes" are present in the upstream regions of many genes involving sporulation such as sigE and spoIIE, and central metabolism such as adc, sol operon, bdhA and $b d h B$ (Wilkinson et al., 1995; Kolek et al., 2017). To regulate the transcription of these genes, the phosphorylated Spo0A will bind to the "0A boxes" to function. In C. acetobutylicum, there are two pathways for Spo0A activation: one depends on a HK Cac0323, and the other involves two HKs Cac0903 and Cac3319, respectively (Steiner et al., 2011). Individual mutants of the three HKs inactivation showed that the sporulation frequency was decreased by 95-99\% compared to the wild-type strain (Steiner et al., 2011), indicating these HKs have similar characteristics and are essential for spore development. More importantly, inactivation of Cac3319 in C. acetobutylicum was found to give positive phenotypic changes, including increased butanol tolerance and production (Xu et al., 2015). However, no relevant study has been performed in C. beijerinckii, another important butanol-producing strain that could more preferably utilize lignocellulosic hydrolysate (Xiao et al., 2012). There are $85 \mathrm{HKs}$ in the genome of C. beijerinckii and only one of them has been investigated to explore its regulatory function. The three-component system consists of
HK LytS and response regulator YesN, which directly regulates the transcription of $x y l F G H$ genes and is responsible for xylose transportation (Sun et al., 2015). However, whether orphan HKs could regulate sporulation and solventogenesis in C. beijerinckii, as they did in C. acetobutylicum, was still unknown. The genome sequence data indicated that clostridia largely shared the key HK genes for phosphorylation of Spo0A (Al-Hinai et al., 2015), and thus we hypothesized that analogous HKs existed and were functional in C. beijerinckii.

In this study, we reported the identification and verification of orphan HKs in C. beijerinckii for their regulatory function in solventogenesis and sporulation. Six HK candidates were identified by sequence alignment with cac3319, cac0323, and cac0903 in C. acetobutylicum. Using CRISPR-Cas9n system, we successfully deleted these HK genes, and fermentation analysis showed that two of them were important for regulating butanol biosynthesis and spore development. This study demonstrated the role of $\mathrm{HKs}$ on sporulation and solventogenesis in C. beijerinckii for the first time. The HK-engineered strains can be used as robust workhorses for enhanced butanol production, and also can be further engineered for production of valuable metabolites.

\section{MATERIALS AND METHODS}

\section{Bacterial Strains, Culture Conditions, and Plasmids}

All bacterial strains and plasmids used in this study were listed in Supplementary Table S1. The Escherichia coli DH5 $\alpha$ was used for plasmid cloning. The E. coli transformants were grown aerobically at $37^{\circ} \mathrm{C}$ in Luria-Bertani (LB) medium or on solid LB agar $(2 \% \mathrm{w} / \mathrm{v})$ plate supplemented with ampicillin $(100 \mu \mathrm{g} / \mathrm{mL})$ when necessary. C. beijerinckii CC101 was an adaptive mutant of C. beijerinckii NCIMB 8052 (ATCC 51743) from ST Yang's lab (Lu et al., 2013), and its mutant strains were grown anaerobically at $37^{\circ} \mathrm{C}$ in Clostridium Growth Medium (CGM) (Li et al., 2016) supplemented with erythromycin $(40 \mu \mathrm{g} / \mathrm{mL})$ as necessary.

\section{Reagents and Enzymes}

All restriction enzymes used in this study were purchased from New England Biolabs (Beverly, MA, United States). The DNA polymerase KOD FX (Toyobo, Osaka, Japan) was used for DNA amplification and colony extension PCR to screen the positive transformants. Recombinant plasmids were assembled through the ClonExpress One Step Cloing Kit (Vazyme Biotech, Nanjing, China).

\section{Plasmid Construction}

All the DNA oligonucleotides used in this study were synthesized in Sangon Biotech Co., Ltd. (Shanghai, China) and listed in Supplementary Table S2. The schematic diagram of the plasmid construction and gene-editing process was shown in Supplementary Figure S1. CRISPR-based genome editing plasmid, pNICKclos 2.0-xylR (Li et al., 2016), was used as the vector to delete $H K$ genes. As the selected target region (N20NGG) was crucial for sgRNA targeting, alignment research (NCBI BLAST) was performed to ensure that the 23-bp 
target sequence had no sequence similarity elsewhere in the genome and thus could avoid multiple off-target candidates. The sgRNA-encoding region targeting the cbei2073 was placed following the Pj23119 synthetic promoter through the steps described below. The primers pNICKclos-2073-1-1/pNICKclos2073-3 were used to generate a Pj23119-sgRNA-2073-1 cassette from pNICKclos 2.0-xylR and then the cassette was used as the template to PCR amplify the Pj23119-sgRNA-2073-2 cassette with primers pNICKclos-2073-1-2/pNICKclos-2073-3. The $1.2-\mathrm{kb}$ upstream and $1.2-\mathrm{kb}$ downstream homology arms (HAs) of the selected target region (N20-NGG) in cbei2073 were amplified from the genome of C. beijerinckii CC101 using primers pairs pNICKclos-2073-2/pNICKclos-2073-5 and pNICKclos-2073-4/pNICKclos-2073-6, respectively. Then the Pj23119-sgRNA-2073-2 cassette and the two HAs were joined by overlap extension PCR using primers pNICKclos-2073-12/pNICKclos-2073-6, generating a fragment in which the two HAs were separated by a PstI restriction site. Finally, the fragment was fused with the SpeI/XhoI linearized pNICKclos 2.0-xylR plasmid through the One Step Cloning Kit, yielding pNICKclos 2.0-cbei2073. All the other plasmids (pNICKclos 2.0-cbei2087, pNICKclos 2.0-cbei2435, pNICKclos 2.0-cbei1553, pNICKclos 2.0-cbei4925, and pNICKclos 2.0-cbei4484) used for gene deletion based on CRISPR-Cas9 nickase in C. beijerinckii CC101 were also derived from pNICKclos 2.0- $x y l R$ using similar methods as described above.

\section{Transformation of C. beijerinckii}

Plasmids for genome editing were transformed into C. beijerinckii via electroporation (Mermelstein et al., 1992) according to the following protocol. To prepare electrocompetent cells, $2 \mathrm{~mL}$ stock culture in $20 \%$ glycerol was inoculated into $100 \mathrm{~mL}$ CGM medium until the optical density at $600 \mathrm{~nm}\left(\mathrm{OD}_{600}\right)$ reached 0.4-0.6. All of the following steps were done at low temperatures $\left(4^{\circ} \mathrm{C}\right)$ and the buffers used were pre-cooled. The culture was centrifuged at $4500 \mathrm{r} / \mathrm{min}$ for $10 \mathrm{~min}$ and then the cells were resuspended in $30 \mathrm{~mL}$ ETM buffer $(270 \mathrm{mM}$ sucrose, $4.4 \mathrm{mM} \mathrm{NaH} \mathrm{PO}_{4}, 0.6 \mathrm{mM} \mathrm{Na}_{2} \mathrm{HPO}_{4}, 10 \mathrm{mM} \mathrm{MgCl}$, $\mathrm{pH}$ 7.4) for $10 \mathrm{~min}$. The resuspended cells were centrifuged

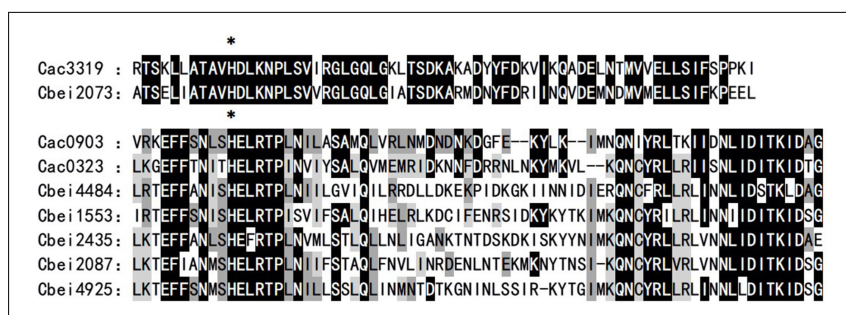

FIGURE 1 | Comparison of HK phosphodonor active site domains. The $\mathrm{DHp} /$ HisKA domains of several putative orphan HKs in C. beijerinckii were compared to known orphan kinases Cac3319, Cac0903, and Cac0323 (C. acetobutylicum), respectively. Identical residues conserved with Cac3319 or Cac0903 and Cac0323 are shaded black, identical residues conserved with Cac0903 are shaded dark gray, and identical residues conserved with $\mathrm{Cac0323}$ are shaded light gray. The phosphorylated histidine residue is denoted by an asterisk. and resuspended in $2.5 \mathrm{ml}$ ET buffer $(270 \mathrm{mM}$ sucrose, $4.4 \mathrm{mM} \mathrm{NaH} \mathrm{PO}_{4}, 0.6 \mathrm{mM} \mathrm{Na} \mathrm{HPO}_{4}, \mathrm{pH}$ 7.4). The mixture of $190 \mu \mathrm{L}$ competent cells and $10 \mu \mathrm{L}$ plasmid DNA (1-2 $\mu \mathrm{g})$ was transferred to an electroporation cuvette with a $0.2 \mathrm{~cm}$ gap width. Electroporation was performed using a Bio-Rad Micropulser (Bio-Rad Laboratories, Hercules, CA, United States) at $1.8 \mathrm{kV}$. After electroporation, samples were mixed with $400 \mu \mathrm{L}$ CGM immediately and transferred to another $400 \mu \mathrm{L}$ $\mathrm{CGM}$ in a 2-mL tube. After incubation for $4-6 \mathrm{~h}$ at $37^{\circ} \mathrm{C}$, $200 \mu \mathrm{L}$ recovered cultures were plated on CGM agar containing $40 \mu \mathrm{g} / \mathrm{mL}$ erythromycin and grown anaerobically at $37^{\circ} \mathrm{C}$ for $48 \mathrm{~h}$. Transformant colonies were picked from the agar plates and subjected to PCR-based verification.

\section{Mutant Screening}

Individual transformant colonies were all picked and analyzed using colony-PCR after electroporation. Then the PCR productions were digested by restriction endonuclease and sequenced to confirm the positive transformant colonies. The primers used were selected from sequence located about $100 \mathrm{bp}$ up- and down-stream of the HAs on the genome named cbei2073-For/cbei2073-Rev, etc. The restriction site (PstI) between the two HAs in the editing template was confirmed by cleavage of the fragment amplified by colony extension PCR with PstI restriction enzyme. Parent strain C. beijerinckii CC101 genome was used as a negative control.

\section{Plasmid Curing}

To remove the pNICKclos 2.0 plasmids from mutants, the positive transformants were cultured in $5 \mathrm{~mL}$ of CGM liquid medium (M1) without antibiotic pressure at first. After growing for $12 \mathrm{~h}, 50 \mu \mathrm{L}$ of $\mathrm{M} 1$ was inoculated into $5 \mathrm{~mL}$ CGM medium without antibiotic (M2). After another $12 \mathrm{~h}$, the $\mathrm{OD}_{600}$ of M2 increased to $\sim 0.8$. Then $100 \mu \mathrm{L}$ of the M2 broth was spread on a CGM medium ager plate without antibiotic. After $12 \mathrm{~h}$, the individual colonies were spread onto CGM ager with erythromycin $(50 \mu \mathrm{g} / \mathrm{mL})$ and CGM ager without antibiotic. The cells that grew on the later but could not grow on the former were considered to have lost the plasmid.

\section{Batch Fermentation}

Batch fermentations with various engineered $C$. beijerinckii strains were performed anaerobically in P2 medium in a 3$\mathrm{L}$ bioreactor with $1.5-\mathrm{L}$ working volume. The P2 medium was prepared as previously described (Xue et al., 2016), which contained glucose $\sim 90 \mathrm{~g} / \mathrm{L}$; yeast extract $1 \mathrm{~g} / \mathrm{L} ; \mathrm{CH}_{3} \mathrm{COONH}_{4}$ $2.2 \mathrm{~g} / \mathrm{L} ; \mathrm{K}_{2} \mathrm{HPO}_{4} 0.5 \mathrm{~g} / \mathrm{L} ; \mathrm{KH}_{2} \mathrm{PO}_{4} 0.5 \mathrm{~g} / \mathrm{L} ; \mathrm{MgSO}_{4} \cdot 7 \mathrm{H}_{2} \mathrm{O}$ $0.2 \mathrm{~g} / \mathrm{L} ; \mathrm{MnSO}_{4} \cdot \mathrm{H}_{2} \mathrm{O} 0.01 \mathrm{~g} / \mathrm{L} ; \mathrm{FeSO}_{4} \cdot 7 \mathrm{H}_{2} \mathrm{O} 0.01 \mathrm{~g} / \mathrm{L} ; \mathrm{NaCl}$ $0.01 \mathrm{~g} / \mathrm{L}$; para-amino-benzoic acid $1 \mathrm{mg} / \mathrm{L}$; thiamin $1 \mathrm{mg} / \mathrm{L}$; and biotin $1 \mathrm{mg} / \mathrm{L}$. The $C$. beijerinckii strains were first incubated anaerobically in $100 \mathrm{~mL}$ of $\mathrm{CGM}$ at $37^{\circ} \mathrm{C}$ until $\mathrm{OD}_{600}$ reached $\sim 1.0$ and then the seed culture was transferred into the bioreactor containing 1.4 L P2 medium. All the media used in this study were sterilized at $121^{\circ} \mathrm{C}$ and 15 psig for $15 \mathrm{~min}$ and purged with $\mathrm{N}_{2}$ for $20 \mathrm{~min}$ through a sterile $0.2 \mu \mathrm{m}$ filter to remove dissolved oxygen. 

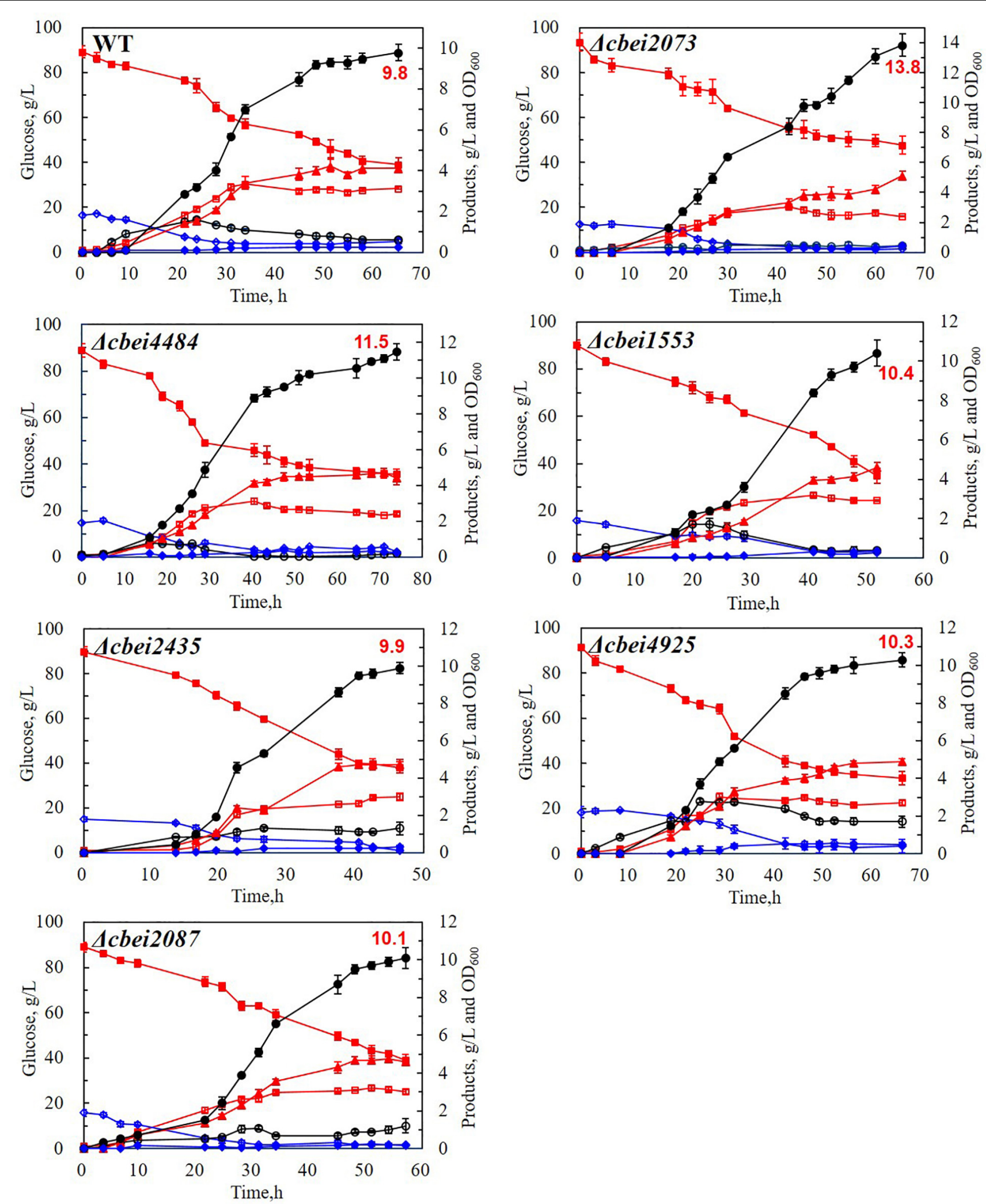

FIGURE 2 | Fermentation results of the wild-type (WT) and mutant strains in batch fermentation. Symbols: glucose (filled squares), OD600 (open squares), butanol (filled circles), acetone (filled triangles), ethanol (filled diamonds); butyrate (open circles), acetate (open diamonds). 


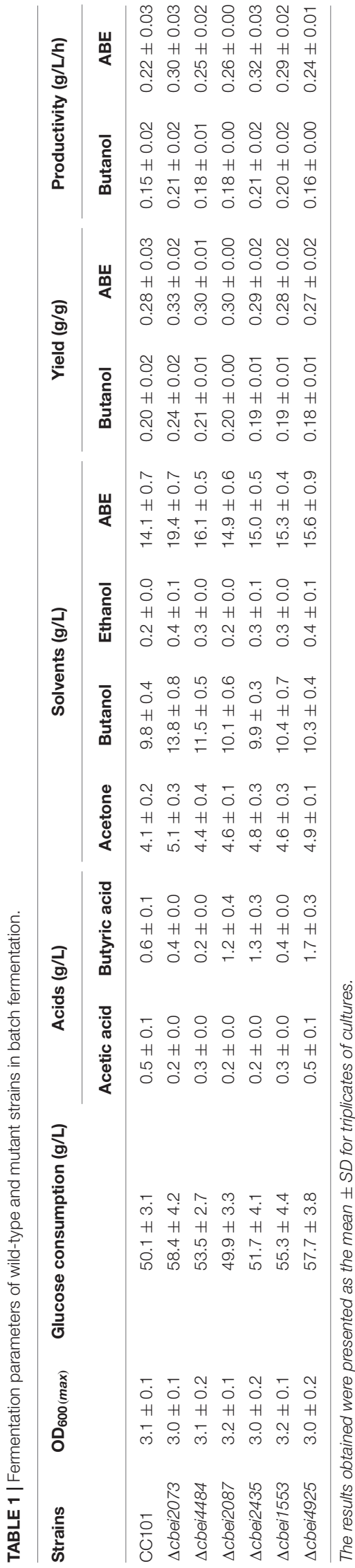

\section{Sporulation Frequency Assay}

The strains were cultured in liquid CGM medium for 5 days. Then the cell cultures were treated in $80^{\circ} \mathrm{C}$ for $10 \mathrm{~min}$, after which $100 \mu \mathrm{L}$ of them were plated on CGM agar directly or following diluting for 10 times. The sporulation frequency of strains was characterized by the numbers of heatresistant colonies.

\section{Analytical Methods}

The fermentation samples were periodically taken from the bioreactor and the cell growth was analyzed by measuring $\mathrm{OD}_{600}$ using a spectrophotometer (Thermo Spectronic, United States). Samples for glucose and product analyses were pelleted by centrifugation at $8000 \times g$ for no less than $5 \mathrm{~min}$. The solvents of the fermentation broth (acetone, butanol, and ethanol) were analyzed using a gas chromatograph (Agilent 6890A GC, United States) as previously described (Xue et al., 2017b; Yang et al., 2019). Glucose, acetate and butyrate were analyzed by HPLC (Waters 1525, United States) equipped with an Aminex HPX-87H column $(300 \mathrm{~mm} \times 7.8 \mathrm{~mm})$ maintained at $50^{\circ} \mathrm{C}$. Dilute $\mathrm{H}_{2} \mathrm{SO}_{4}(10 \mathrm{mM}, 0.5 \mathrm{~mL} / \mathrm{min})$ was used as the eluent (Xiao et al., 2019).

\section{RESULTS}

\section{Screening of Target Genes and Construction of Engineered Strains}

To explore the uncharacterized HKs in C. beijerinckii, sequence alignments between the nucleotide sequence of three orphan HK genes in C. acetobutylicum (cac3319, cac0323, and cac0903) and the whole genome of $C$. beijerinckii were performed. Six candidates, including cbei2073, cbei2087, cbei4925, cbei2435, cbei1553, and cbei4484, were identified. Among them, cbei2073 showed 46\% homology with cac3319, and the others showed $31-36 \%$ homology with cac0323 or cac0903 gene. By further amino acid sequence alignment of HisKA domain, Cbei2073 showed 78\% sequence similarity with Cac3319 and the others showed no less than $70 \%$ with Cac0903 or Cac0323. Also, Cbei2073 was special among the six in that its phosphorylation sites and their adjacent regions were highly conserved and similar to Cac3319, which has been proved to be the most functional $\mathrm{HK}$ in regulating butanol biosynthesis in C. acetobutylicum (Figure 1; Xu et al., 2015). Based on these analyses, these six HKs were finally selected as candidate homologous proteins.

After selecting these targeting genes, recombinant plasmids (pNICKclos 2.0-cbei2073, pNICKclos 2.0-cbei2087, pNICKclos 2.0-cbei2435, pNICKclos 2.0-cbei1553, pNICKclos 2.0-cbei4925, and pNICKclos 2.0-cbei4484) were first constructed to delete the corresponding gene, respectively. Then they were separately transferred into C. beijerinckii via electroporation. A low number of transformants could been identified after electroporation, but the verification results showed that the accuracy rates (number of correctly edited transformants/total number of transformants screened) were no less than 60\% (Supplementary Table S3). 


\section{Impact of HK Genes on ABE Fermentation}

To investigate the effect of HKs on solventogenesis and cell growth, batch fermentation performances of wild-type C. beijerinckii and HK-inactivated strains were compared (Figure 2 and Table 1). Cbei2073 inactivated strain showed enhanced butanol production with 40.8 and $40.0 \%$ increases in butanol titer and butanol productivity, respectively, compared to wild-type C. beijerinckii. In addition, increases in ethanol and acetone were also observed in Cbei2073 inactivated strain. According to the fermentation kinetics study, it could be concluded that Cbei2073 played an important role in regulating solvent production, which was similar to $\mathrm{HK}$ Cac3319 of C. acetobutylicum. Cbei4484 inactivated strain showed enhanced butanol production with 17.3 and 20.0\% increases in butanol titer and butanol productivity, respectively. Fermentation of Cbei1553 mutant strain showed that this HK had minor effects on cell growth and solvent production, but its inactivation resulted in $40.0 \%$ increase in butanol productivity. The ABE titers of Cbei2435, Cbei2087, and Cbei4925 inactivated strains were similar to that of the wild strain. However, Cbei2435 and Cbei2087 mutant strains showed slight increases in butyrate titer and significant increases in butanol productivity.

\section{Effect of HKs on Sporulation Frequency}

As spore-forming cells were metabolically inactive, it is hypothesized that the enhanced butanol production was resulted from reduced sporulation frequency caused by inactivation of HK. The heat-resistant colonies of all the six HK mutants and wild-type strain were tested. It was found that the heatresistant colonies of four HK genes (cbei2435, cbei1553, cbei2087, and Cbei4925) deleted strains were in the same level with wild-type strain, which were much more than the other two mutants ( $\Delta c b e i 2073$ and $\Delta c b e i 4484)$. Since cbei2073 and cbei4484 had obvious effect on butanol production, we compared the sporulation frequency of wild-type strain, cbei2073 and cbei4484 deleted strains by counting the number of heat-resistant colonies following incubation in liquid CGM medium for 5 days. As excepted, significant decreases of sporulation frequency were observed for two HKs inactivated strains, with spore formation reduced by 96.9 and $77.4 \%$ of the wild-type strain, respectively (Figure 3). It was indicated that these two HKs might have effects on sporulation and its related genes.

\section{DISCUSSION}

CRISPR-Cas9n based gene editing technology is a method which is not only effective but also widely used in bacteria including C. acetobutylicum, C. beijerinckii, Bacillus licheniformis, etc. (Wang et al., 2015, 2016a,b; Li et al., 2016, 2018; Zhang et al., 2018). Despite the wide application of this system, it remains reliant on homologous recombination (HR) (Li et al., 2016). In clostridia, HR is notoriously inefficient, which cannot be solved by CRISPR-Cas9 technologies alone (Bruder et al., 2016; Charubin et al., 2018). For this reason, the numbers of individual transformant colonies obtained after the transformation of plasmids into C. beijerinckii and the edited cells were both small (Supplementary Table S3). However, the verification results showed that the accuracy rates (60.0-100.0\%) were relative higher than those (6.7-100.0\%) obtained in a previous study (Li et al., 2016).

Orphan HKs in Clostridium species are reported to directly phosphorylate Spo0A, which is the master regulator for initiation of sporulation and solventogenesis (Steiner et al., 2011; Dürre, 2014). To explore the regulatory function of HKs on biobutanol synthesis, the $85 \mathrm{HK}$ genes in C. beijerinckii were analyzed and compared with three HKs (Cac3319, Cac0903, and Cac0323) in C. acetobutylicum with elucidated functions. Cac3319 inactivated strains could produce $44.4 \%$ more butanol ( $\mathrm{Xu}$ et al., 2015), indicating its vital role in regulating solvent production in C. acetobutylicum. In this study, Cac3319 and Cbei2073 were
A

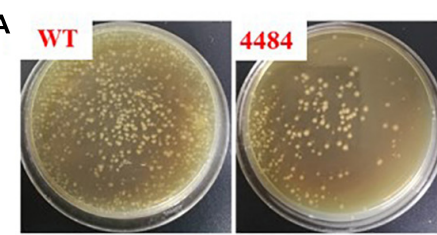

(a)

(c)
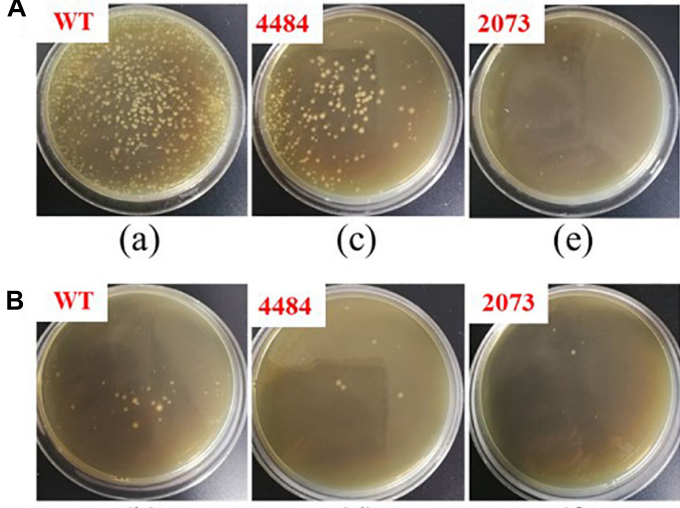

(b) (e)

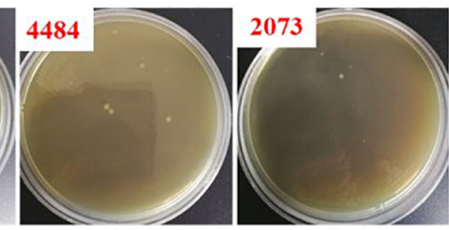

(d)

(f)

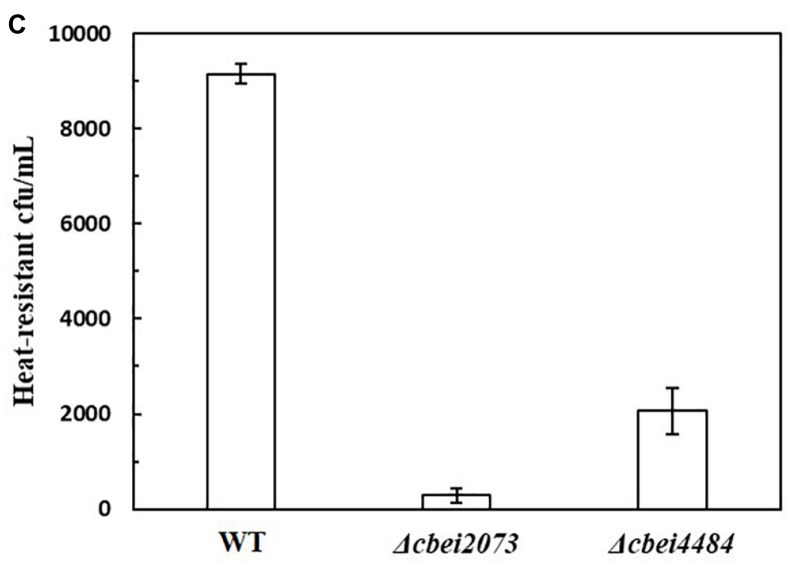

FIGURE 3 | Sporulation frequencies of the wild-type (WT) and mutant strains. The heat-resistant colony-forming units (CFU) were evaluated by treating the cell cultures (5 days) in $80^{\circ} \mathrm{C}$ for $10 \mathrm{~min}$, after which $100 \mu \mathrm{L}$ of them were plated on CGM agar directly (A) or following diluting for 10 times (B). (C) The numbers of heat-resistant colonies. 
similar based on the sequence alignment results. The deletion of cbei2073 gene led to significant change in butanol biosynthesis with butanol production increased by $40.8 \%$, indicating that Cbei2073 was also most effective in regulating biobutanol synthesis among the selected six HKs. However, some HKs including Cbei1553, Cbei4925, Cbei2435, and Cbei2087 seemed to have no apparent effect on ABE synthesis, and the growth of all the strains with inactivated HKs was not affected.

In C. acetobutylicum, sporulation is closely associated with solventogenesis, and both of them are controlled by Spo0A (Steiner et al., 2011). Cac3319, Cac0903, and Cac0323 are essential for Spo0A activation, which controls the initiation of sporulation and solventogenesis (Steiner et al., 2011; Xu et al., 2015). The "0A box" that can be bound with Spo0A P has been proved present in the upstream regions of lots of genes whose expressions are either activated or repressed at the initiation of solventogenesis in both C. acetobutylicum and C. beijerinckii (Wilkinson et al., 1995). Generally, the sporulating phenotype is considered to be profitable for solvent formation, but solvent production ceases when mature spores form (Kolek et al., 2016). In addition, overexpression of spo0A in C. beijerinckii NRRL B-598, sharing high genome homology with C. beijerinckii NCIMB 8052, led to cessation of production at a low $\mathrm{ABE}$ concentration, indicating that $\mathrm{Spo} 0 \mathrm{~A}$ is a master regulator of solventogenesis in $C$. beijerinckii (Kolek et al., 2017). Furthermore, without spore formation, C. beijerinckii NRRL B-598 could produce more butanol in RCM medium, compared with the sporulating phenotype (Branska et al., 2018). Therefore, we speculated that Cbei2073 and Cbei4484 played a role on phosphorylation of Spo0A due to their high similarity in sequence with Cac3319, Cac0903, and Cac0323 and further regulated both butanol synthesis and sporulation frequency. As shown in the results, the inactivation of the two HKs (Cbei2073 and Cbei4484) may lower Spo0A P level and then decrease sporulation frequency, finally leading to the improved butanol production. Selective inhibition of sporulation process was beneficial for improving the economics of $\mathrm{ABE}$ fermentation since solvent can be produced during a longer time frame (Cheng et al., 2019).

In conclusion, the results of the present study demonstrated that by deleting $\mathrm{HK}$ genes, the sporulation frequency might be decreased, with different degrees of improvements in butanol titers, productivities and yields (Table 1). Therefore, this study provided a novel strategy for promoting production of metabolites applicable for a broad of bacteria.

\section{REFERENCES}

Al-Hinai, M. A., Jones, S. W., and Papoutsakis, E. T. (2015). The Clostridium sporulation programs: diversity and preservation of endospore differentiation. Microbiol. Mol. Biol. Rev. 79, 19-37. doi: 10.1128/MMBR.000 $25-14$

Branska, B., Pechacova, Z., Kolek, J., Vasylkivska, M., and Patakova, P. (2018). Flow cytometry analysis of Clostridium beijerinckii NRRL B-598 populations exhibiting different phenotypes induced by changes in cultivation conditions. Biotechnol. Biofuels 11:99. doi: 10.1186/s13068-018-1096-x

Bruder, M. R., Pyne, M. E., Moo-Young, M., Chung, D. A., and Chou, C. P. (2016). Extending CRISPR-Cas9 technology from genome editing to transcriptional

\section{DATA AVAILABILITY STATEMENT}

The raw data supporting the conclusions of this article will be made available by the authors, without undue reservation, to any qualified researcher.

\section{AUTHOR CONTRIBUTIONS}

CX developed the research scheme. XX performed the experiments and drafted the manuscript. XX, CC, GD, and CX were involved in the data interpretation and result discussion. $\mathrm{XX}, \mathrm{CC}, \mathrm{GD}, \mathrm{LC}$, and CX were involved in the manuscript revision. All authors read and approved the final manuscript.

\section{FUNDING}

This work was supported by the National Key R\&D Program of China (2018YFB1501703), the National Natural Science Foundation of China (NSFC) with Grant number (21878035 and 21576045), the Liaoning Revitalization Talents Program (XLYC1807269), the Dalian Science and Technology Innovation Project (2018J12SN074), the Liaoning Innovative Talent Support Program (LR2017005), the Youth Science and Technology Star Project of Dalian (2017RQ003), the Talent Cultivation Plan of "Xinghai Scholar" from Dalian University of Technology, and the Fundamental Research Funds for the Central Universities (DUT19ZD213).

\section{ACKNOWLEDGMENTS}

We would like to thank Prof. Shang-Tian Yang (The Ohio State University, United States) for donating the strain C. beijerinckii CC101 and Prof. Sheng Yang and Prof. Weihong Jiang (Shanghai Institutes for Biological Sciences, China) for providing the vector pNICKclos 2.0- $x y l R$ used for genome editing.

\section{SUPPLEMENTARY MATERIAL}

The Supplementary Material for this article can be found online at: https://www.frontiersin.org/articles/10.3389/fbioe. 2020.00214/full\#supplementary-material

engineering in the genus Clostridium. Appl. Environ. Microbiol. 82, 6109-6119. doi: 10.1128/AEM.02128-16

Charubin, K., Bennett, R. K., Fast, A. G., and Papoutsakis, E. T. (2018). Engineering Clostridium organisms as microbial cell-factories: challenges \& opportunities. Metab. Eng. 50, 173-191. doi: 10.1016/j.ymben.2018.07.012

Cheng, C., Bao, T., and Yang, S. T. (2019). Engineering Clostridium for improved solvent production: recent progress and perspective. Appl. Environ. Microbiol. 103, 5549-5566. doi: 10.1007/s00253-019-09916-7

Dürre, P. (2014). Physiology and sporulation in Clostridium. Microbiol. Spectr. 2:4. doi: 10.1128/microbiolspec.TBS-0010-2012

Herman, N. A., Kim, S. J., Li, J. S., Cai, W., Koshino, H., and Zhang, W. (2017). The industrial anaerobe Clostridium acetobutylicum uses polyketides to regulate 
cellular differentiation. Nat. Commun. 8:1514. doi: 10.1038/s41467-01701809-5

Hiscox, T. J., Harrison, P. F., Chakravorty, A., Choo, J. M., Ohtani, K., Shimizu, T., et al. (2013). Regulation of sialidase production in Clostridium perfringens by the orphan sensor histidine kinase ReeS. PLoS One 8:e73525. doi: 10.1371/ journal.pone.0073525

Jacobs, C., Domian, I. J., Maddock, J. R., and Shapiro, L. (1999). Cell cycledependent polar localization of an essential bacterial histidine kinase that controls DNA replication and cell division. Cell 97, 111-120. doi: 10.1016/ S0092-8674(00)80719-9

Kolek, J., Branska, B., Drahokoupil, M., Patakova, P., and Melzoch, K. (2016). Evaluation of viability, metabolic activity and spore quantity in clostridial cultures during ABE fermentation. FEMS Microbiol. Lett 363:fnw031. doi: 10. 1093/femsle/fnw031

Kolek, J., Diallo, M., Vasylkivska, M., Branska, B., Sedlar, K., López-Contreras, A. M., et al. (2017). Comparison of expression of key sporulation, solventogenic and acetogenic genes in C. beijerinckii NRRL B-598 and its mutant strain overexpressing spo0A. Appl. Microbiol. Biotechnol. 101, 8279-8291. doi: 10. 1007/s00253-017-8555-3

Li, K., Cai, D., Wang, Z., He, Z., and Chen, S. (2018). Development of an efficient genome editing tool in Bacillus licheniformis using CRISPR-Cas9 nickase. Appl. Environ. Microbiol. 84, e2608-e2617. doi: 10.1128/AEM.02608-17

Li, Q., Chen, J., Minton, N. P., Zhang, Y., Wen, Z., Liu, J., et al. (2016). CRISPR-based genome editing and expression control systems in Clostridium acetobutylicum and Clostridium beijerinckii. Biotechnol. J. 11, 961-972. doi: 10.1002/biot.201600053

Li, S. Y., Srivastava, R., Suib, S. L., Li, Y., and Parnas, R. S. (2011). Performance of batch, fed-batch, and continuous A-B-E fermentation with pH-control. Bioresour. Technol. 102, 4241-4250. doi: 10.1016/j.biortech.2010. 12.078

Loomis, W. F., Shaulsky, G., and Wang, N. (1997). Histidine kinases in signal transduction pathways of eukaryotes. J. Cell. Sci. 110, 1141-1145.

Lu, C., Dong, J., and Yang, S. T. (2013). Butanol production from wood pulping hydrolysate in an integrated fermentation-gas stripping process. Bioresour. Technol. 143, 467-475. doi: 10.1016/j.biortech.2013.06.012

Mearls, E. B., and Lynd, L. R. (2014). The identification of four histidine kinases that influence sporulation in Clostridium thermocellum. Anaerobe 28, 109-119. doi: 10.1016/j.anaerobe.2014.06.004

Mermelstein, L. D., Welker, N. E., Bennett, G. N., and Papoutsakis, E. T. (1992). Expression of cloned homologous fermentative genes in Clostridium acetobutylicum ATCC 824. Nat. Biotechnol. 10, 190-195. doi: 10.1038/nbt0 292-190

Steiner, E., Dago, A. E., Young, D. I., Heap, J. T., Minton, N. P., and Young, M. (2011). Multiple orphan histidine kinases interact directly with Spo0A to control the initiation of endospore formation in Clostridium acetobutylicum. Mol. Microbiol. 80, 641-654. doi: 10.1111/j.1365-2958.2011.07608.x

Sun, Z., Chen, Y., Yang, C., Yang, S., Gu, Y., and Jiang, W. (2015). A novel three-component system-based regulatory model for $\mathrm{d}$-xylose sensing and transport in Clostridium beijerinckii. Mol. Microbiol. 95, 576-589. doi: 10.1111/ mmi.12894

Underwood, S., Guan, S., Vijayasubhash, V., Baines, S. D., Graham, L., Lewis, R. J., et al. (2009). Characterization of the sporulation initiation pathway of Clostridium difficile and its role in toxin production. J. Bacteriol. 191, 72967305. doi: 10.1128/JB.00882-09

Wang, Y., Zhang, Z. T., Seo, S. O., Choi, K., Lu, T., Jin, Y. S., et al. (2015). Markerless chromosomal gene deletion in Clostridium beijerinckii using CRISPR/Cas9 system. J. Biotechnol. 200, 1-5. doi: 10.1016/j.jbiotec.2015.02.005

Wang, Y., Zhang, Z. T., Seo, S. O., Lynn, P., Lu, T., Jin, Y. S., et al. (2016a). Gene transcription repression in Clostridium beijerinckii using CRISPR-dCas9. Biotechnol. Bioeng. 113, 2739-2743. doi: 10.1002/bit.26020

Wang, Y., Zhang, Z. T., Seo, S. O., Lynn, P., Lu, T., Jin, Y. S., et al. (2016b). Bacterial genome editing with CRISPR-Cas9: deletion, integration, single nucleotide modification, and desirable "clean" mutant selection in Clostridium beijerinckii as an example. ACS Synth. Biol. 5, 721-732. doi: 10.1021/acssynbio.6b00060
Wilke, K. E., Francis, S., and Carlson, E. E. (2015). Inactivation of multiple bacterial histidine kinases by targeting the ATP-binding domain. ACS Chem. Biol. 10, 328-335. doi: 10.1021/cb5008019

Wilkinson, S. R., Young, D. I., Gareth Morris, J., and Young, M. (1995). Molecular genetics and the initiation of solventogenesis in Clostridium beijerinckii (formerly Clostridium acetobutylicum) NCIMB 8052. FEMS Microbiol. Rev. 17, 275-285. doi: 10.1111/j.1574-6976.1995.tb00211.x

Wörner, K., Szurmant, H., Chiang, C., and Hoch, J. A. (2006). Phosphorylation and functional analysis of the sporulation initiation factor Spo0A from Clostridium botulinum. Mol. Microbiol. 59, 1000-1012. doi: 10.1111/j.1365-2958.2005. 04988.x

Xiao, H., Li, Z., Jiang, Y., Yang, Y., Jiang, W., and Gu, Y. (2012). Metabolic engineering of D-xylose pathway in Clostridium beijerinckii to optimize solvent production from xylose mother liquid. Metab. Eng. 14, 569-578. doi: 10.1016/j. ymben.2012.05.003

Xiao, M., Wang, L., Wu, Y., Cheng, C., Chen, L., and Chen, H. (2019). Hybrid dilute sulfuric acid and aqueous ammonia pretreatment for improving butanol production from corn stover with reduced wastewater generation. Bioresour. Technol. 278, 460-463. doi: 10.1016/j.biortech.2019.01.079

Xu, M., Zhao, J., Yu, L., Tang, I. C., Xue, C., and Yang, S. T. (2015). Engineering Clostridium acetobutylicum with a histidine kinase knockout for enhanced n-butanol tolerance and production. Appl. Microbiol. Biotechnol. 99, 10111022. doi: 10.1007/s00253-014-6249-7

Xue, C., and Cheng, C. (2019). "Butanol production by Clostridium," in Advances in Bioenergy, ed. A. Jackman (San Diego, CA: Academic Press), 35-77. doi: 10.1016/bs.aibe.2018.12.001

Xue, C., Liu, F., Xu, M., Zhao, J., Chen, L., Ren, J., et al. (2016). A novel in situ gas stripping-pervaporation process integrated with acetone-butanol-ethanol fermentation for hyper n-butanol production. Biotechnol. Bioeng. 113, 120-129. doi: 10.1002/bit.25666

Xue, C., Zhao, J., Chen, L., Yang, S. T., and Bai, F. (2017a). Recent advances and state-of-the-art strategies in strain and process engineering for biobutanol production by Clostridium acetobutylicum. Biotechnol. Adv. 35, 310-322. doi: 10.1016/j.biotechadv.2017.01.007

Xue, C., Liu, M., Guo, X., Hudson, E. P., Chen, L., Bai, F., et al. (2017b). Bridging chemical-and bio-catalysis: high-value liquid transportation fuel production from renewable agricultural residues. Green Chem. 19, 660-669. doi: 10.1039/ C6GC02546C

Xue, C., Zhao, X. Q., Liu, C. G., Chen, L. J., and Bai, F. W. (2013). Prospective and development of butanol as an advanced biofuel. Biotechnol. Adv. 31, 1575-1584. doi: 10.1016/j.biotechadv.2013.08.004

Yang, D., Cheng, C., Bao, M., Chen, L., Bao, Y., and Xue, C. (2019). The pervaporative membrane with vertically aligned carbon nanotube nanochannel for enhancing butanol recovery. J. Membr. Sci. 577, 51-59. doi: 10.1016/j. memsci.2019.01.032

Yang, D., Tian, D., Xue, C., Gao, F., Liu, Y., Li, H., et al. (2018). Tuned fabrication of the aligned and opened CNT membrane with exceptionally high permeability and selectivity for bioalcohol recovery. Nano Lett. 18, 6150-6156. doi: 10.1021/ acs.nanolett.8b01831

Zhang, Z. T., Jiménez-Bonilla, P., Seo, S. O., Lu, T., Jin, Y. S., Blaschek, H. P., et al. (2018). "Bacterial genome editing with CRISPR-Cas9: taking Clostridium beijerinckii as an example," in Synthetic Biology, ed. J. Braman (New York, NY: Humana Press), 297-325. doi: 10.1007/978-1-4939-7795-6_17

Conflict of Interest: The authors declare that the research was conducted in the absence of any commercial or financial relationships that could be construed as a potential conflict of interest.

Copyright (c) 2020 Xin, Cheng, Du, Chen and Xue. This is an open-access article distributed under the terms of the Creative Commons Attribution License (CC BY). The use, distribution or reproduction in other forums is permitted, provided the original author(s) and the copyright owner(s) are credited and that the original publication in this journal is cited, in accordance with accepted academic practice. No use, distribution or reproduction is permitted which does not comply with these terms. 